\title{
p53 inhibits the upregulation of sirtuin 1 expression induced by c-Myc
}

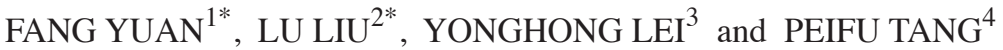 \\ Departments of ${ }^{1}$ Oncology and ${ }^{2}$ Clinical Nutrition, General Hospital of Chinese PLA, Beijing 100853; \\ ${ }^{3}$ Key Laboratory of Wound Repair and Regeneration of The First Affiliated Hospital of Chinese PLA General Hospital, \\ Beijing 100048; ${ }^{4}$ Department of Orthopedics, General Hospital of Chinese PLA, Beijing 100853, P.R. China
}

Received December 7, 2015; Accepted May 16, 2017

DOI: $10.3892 / \mathrm{ol} .2017 .6661$

\begin{abstract}
Sirtuin 1 (Sirt1), a conserved NAD ${ }^{+}$dependent deacetylase, is a mediator of life span by calorie restriction. However, Sirt1 may paradoxically increase the risk of cancer. Accordingly, the expression level of Sirtl is selectively elevated in numerous types of cancer cell; however, the mechanisms underlying the differential regulation remain largely unknown. The present study demonstrated that oncoprotein c-Myc was a direct regulator of Sirt1, which accounts for the upregulation of Sirt1 expression only in the cells without functional p53. In p53 deficient cells, the overexpression of c-Myc increased Sirt1 mRNA and protein expression levels as well as its promoter activity, whereas the inhibitor of c-Myc, 10058-F4, induced decreased Sirt1 basal mRNA and protein expression levels. Deletion/mutation mapping analyses revealed that c-Myc bound to the conserved E-box[-189 to -183 base pair (bp)] of the Sirt1 promoter. In addition, p53 and c-Myc shared at least response element and the presence of p53 may block the binding of c-Myc to the Sirt1 promoter, thus inhibit the c-Myc mediated upregulation of Sirt1 promoter activity. The present study indicated that the expression level of Sirt1 was tightly regulated by oncoprotein c-Myc and tumor suppressor p53, which aids an improved understanding of its expression regulation and tumor promoter role in certain conditions.
\end{abstract}

Correspondence to: Dr Yonghong Lei, Key Laboratory of Wound Repair and Regeneration of The First Affiliated Hospital of Chinese PLA General Hospital, 51 Fucheng Road, Haidian, Beijing 100048, P.R. China

E-mail:1yhhlx@126.com

Dr Peifu Tang, Department of Orthopedics, General Hospital of Chinese PLA, 28 Fuxing Road, Haidian, Beijing 100853, P.R. China E-mail: pftang301@126.com

*Contributed equally

Key words: sirtuin 1, c-Myc, p53, tumorigenesis

\section{Introduction}

Sirtuin 1 (Sirt1), a conserved NAD ${ }^{+}$dependent deacetylase, has been implicated in modulating transcriptional silencing and cell survival, and is known to serve a role in tumorigenesis via deacetylation of important transcriptional factors, including tumor protein p53 (p53) (1), E2F transcription factor 1 (E2F1) (2) and nuclear factor- $\kappa \mathrm{B}(3)$.

Despite the paradoxical role of Sirt1 in tumorigenesis, the expression level of Sirtl is increased in numerous types of cancer cell. Upregulation of Sirt1 is frequently observed in non-melanoma skin cancers, including actinic keratosis, Bowen's disease, squamous cell carcinoma and basal cell carcinoma (4). Sirtl expression level is also significantly increased in poorly differentiated mouse and human prostate cancer (5), acute myeloid leukemia (AML) (6) and lymphoma (7).

The expression level of Sirtl is differentially regulated by oncogenes and tumor suppressor genes. p53 has been reported to repress the expression of Sirtl and its removal by forkhead box $\mathrm{O} 3$ activated Sirt1 transcription in PC12 neuronal cancer cells $(8,9)$. This model is further supported by studies investigating transformation-associated p53 knockout mice, which expressed constitutively higher Sirt1 mRNA expression levels in numerous tissues (10).

Of note, hypermethylated in cancer 1 (HIC1), another tumor suppressor that acts as a repressor of Sirt1 expression level, formed a transcriptional repression complex with Sirt1, which induced inhibition of Sirt1 transcription (11). Sirt1 expression is regulated by the transcriptional factor E2F1, which binds two sites within the Sirt1 promoter. E2F1 is a crucial activator of Sirtl expression level in response to DNA damage (2). Of note, the majority of the identified transcriptional factors associated with Sirtl expression regulation are also subjected to Sirt1 deacetylation and form positive or negative feedback loops to fine-tune cell fate.

c-Myc is another oncogenic transcriptional factor that is deacetylated by Sirt1 (12). It remains unclear whether c-Myc may be involved in feedback loops of Sirt1 deacetylation. In the present study it was revealed that c-Myc directly binds to the conserved E-box (-189 to $183 \mathrm{bp}$ ) of the Sirt1 promoter and induces its transcription in p53 deficient cells. p53 inhibited the c-Myc mediated upregulation of Sirt1 promoter activity by blocking its binding to the Sirtl promoter. This novel 
regulation of Sirt1 identified it as the prototype for a novel class of c-Myc and p53 target genes and aids understanding of its role in certain tumor cells.

\section{Materials and methods}

Cell culture, transfection and, reagents. Mouse embryonic fibroblasts MEF/3T3, human embryonic kidney 293A, human colorectal cancer HCT116, human osteosarcoma U2OS, human leukemic K562 and human non-small cell lung carcinoma H1299 cells were purchased from the American Type Culture Collection (Manassas, VA, USA). All cells were maintained in Dulbecco's modified Eagle's medium (Thermo Fisher Scientific, Inc., Waltham, MA, USA) supplemented with glutamine, penicillin/streptomycin and 10\% fetal bovine serum (Sigma-Aldrich; Merck KGaA, Darmstadt, Germany). All transfections were performed using Lipofectamine ${ }^{\circledR} 2000$ (Invitrogen; Thermo Fisher Scientific, Inc., Waltham, MA, USA), according to the manufacturer's protocol. 10058-F4 was purchased from Sigma-Aldrich (Merck KGaA).

Constructs and antibodies. Flag-tagged c-Myc and human influenza hemagglutinin-tagged p53 expression vectors were provided by Wuhan Sanyinh Biotechnology (Wuhan, China).

For the luciferase reporter assay, various length fragments of the Sirt1 promoter, starting from either-2852, -200 , or -102 to the transcriptional start site $(+1)$ were amplified from human genomic DNA. The primers used in luciferase reporter generation were as follows: -2852 fragment (E1/2/3), 5'-GACTCG AGCGTCAGCCACCGTGCT-3' and 5'-TTGCTAGCTCTT CCAACTGCCTCT-3'; -200 fragment (E2/3), 5'-TTAAGC TTCCTCCGCCCGCCACGT-3' and 5'-TCGGTACCTCTT CCAACTGCCTCT-3'; -102 fragment, 5'-TTAAGCTTGGGT TTAAATCTCCCG-3' and 5'-ATGGTACCTCTTCCAACT GCCTCT-3'. In the - 200 fragment, two putative c-Myc binding sites containing the consensus 'CACGTG' were identified using rVISTA2.0 (https://rvista.dcode.org/). Mutant promoter constructs were obtained by changing each 'CACGTG' sequence within the -200 fragment into 'TTTGGG' and named as E2/mutant (mut) or E3/mut respectively. All amplified promoter fragments were cloned into the pGL3-basic luciferase reporter (Promega Corporation, Madison, WI, USA) within restriction enzyme sites SmaI and HindIII. The scrambled siRNA (control) and siRNAs targeting p53 were purchased from Thermo Fisher Scientific, Inc. Indicated overexpression constructs were generated by amplifying the coding region from cDNA obtained from $\mathrm{MEF} / 3 \mathrm{~T} 3$ and H1299 cells and sub-cloned into the pEF-HA vector (Addgene, Inc., Cambridge, MA, USA). MEF/3T3 and H1299 cells were transiently transfected with siRNAs targeting p53 using Lipofectamine ${ }^{\circledR} 2000$ (Invitrogen; Thermo Fisher Scientific, Inc.), according to the manufacturer's protocol.

Anti-c-Myc (N262; dilution, 1:100; cat. no. sc-500771), anti-human Sirt1 (dilution, 1:200; cat. no. sc-15404), anti-p53 (dilution, 1:500; cat. no. sc-126) and anti-GAPDH (dilution, 1:500; cat. no. sc-47724) antibodies were purchased from Santa Cruz Biotechnology, Inc. (Dallas, TX, USA).

Luciferase reporter assay. $\mathrm{H} 1299$ cells were harvested at $36 \mathrm{~h}$ after transfection. Cellular promoter activity was determined using the Dual Luciferase Assay System kit, according to the manufacturer's protocol (Promega Corporation). pRL-CMV renilla luciferase was used as an internal control.

Reverse transcription-quantitative polymerase chain reaction $(R T-q P C R)$. The expression level of Sirt1 was determined by RT of total RNA followed by qPCR analysis. Total RNA was extracted from K562, 293A and MEF/3T3 cells using an RNA extraction kit (Invitrogen; Thermo Fisher Scientific, Inc.) according to the manufacturer's protocol. A total of $2 \mu \mathrm{g}$ total RNA was reverse transcribed by extension of oligo dT primers using M-MLV reverse transcriptase (New England Biolabs, Inc., Ipswich, MA, USA), according to the manufacturer's protocol. qPCR was performed on a Bio-Rad IQ5 cycler (Bio-Rad Laboratories, Inc., Hercules, CA, USA) using SYBR-Green Master Mix (Takara Biotechnology, Co., Ltd., Dalian, China). The primers used were as follows: Human sirt1, CAGTGGCTGGAACAGTGAGA (forward) and TCT GGCATGTCCCACTATCA (reverse); human GAPDH, GAG TCAACGGATTTGGTCGT (forward) and GACAAGCTT CCCGTTCTCAG (reverse); mouse sirt1, GTAAGCGGCTTG AGGG (forward) and TTCGGGCCTCTCCGTA (reverse); mouse GAPDH, CGTCCCGTAGACAAAATGGT (forward) and GAATTTGCCGTGAGTGGAGT (reverse). The program was as follows: $95^{\circ} \mathrm{C}$ for $2 \mathrm{~min}$, followed by 30 cycles of denaturation at $95^{\circ} \mathrm{C}$ for $5 \mathrm{sec}$, and annealing/extension temperature $\left(55 / 68^{\circ} \mathrm{C}\right)$ for $1 \mathrm{~min}$. GAPDH was used as reference gene and the method of $2^{-\Delta \Delta \mathrm{Cq}}$ was applied for quantification (13).

Chromatin immunoprecipitation (ChIP). K562 and 293A cells were treated with $1 \%$ formaldehyde for $15 \mathrm{~min}$ at room temperature. Cross-linked chromatin was sheared by sonication for 5 cycles ( $30 \mathrm{sec}$ on $/ 30 \mathrm{sec}$ off) at $4^{\circ} \mathrm{C}$ to $500-1,000 \mathrm{bp}$ and then used for IP with anti-cMyc antibodies as previously described (dilution, 1:100) or non-immune rabbit immunoglobulin G (dilution, 1:500; cat. no., 2729S; Cell Signaling Technology, Inc., Danvers, MA, USA) as a negative control. Immunoprecipitated complexes were collected by protein A Sepharose beads (Upstate Biotechnology, Inc., Lake Placid, NY, USA). The immunoprecipitated chromatin was amplified by PCR with DNA polymerase (Takara Biotechnology, Co., Ltd.) using primers for the human telomerase reverse transcriptase (hTERT) or Sirt1 promoter. The primers were as follows: hTERT, 5'-AGGCCGGGCTCCCAGTGGATT C-3' and 5'-CGTGGCCAGCGGCAGCACCTC-3'; Sirt1 E2/3, 5'-GGAGCGGTAGACGCAACA-3' and 5'-CTTCCA ACTGCCTCTCTGG-3'; Sirt1 E1: 5'-AGGCCAAGTCAT TTCCTTCC-3' and 5'-ACCTTTGACGTGGAGGTTTG-3'. The program was as follows: $95^{\circ} \mathrm{C}$ for $5 \mathrm{~min}$, followed by 30 cycles of denaturation at $95^{\circ} \mathrm{C}$ for $5 \mathrm{sec}$, annealing/extension temperature $\left(55 / 68^{\circ} \mathrm{C}\right)$ for $1 \mathrm{~min}$, and a final extension of $68^{\circ} \mathrm{C}$ for $10 \mathrm{~min}$. The resulting PCR products were separated by $2 \%$ agarose gel electrophoresis.

DNA mediated precipitation. The DNA-protein complex was cross-linked and chromatin DNA was sheared by sonication as previously described. A total of $4 \mu \mathrm{g}$ biotinylated double strand oligonucleotide from human Sirtl promoter 222 (5'-CCCAGGCGGAGCGGTAGACGCAACAGCCTCCGCCCG CCACGTGACCCGTA-3') or the control 276 (5'-CGCCAC 
AAAGAGGAAGGGCCGCCGGCCGCCGGGGCCGAG TGCGCTTCCAG-3') were incubated with HeLa cell lysate (Abcam, Cambridge, MA, USA) and $30 \mu 1$ streptavidin agarose beads (BioVision, Inc., Milpitas, CA, USA) slurry at $4^{\circ} \mathrm{C}$ for $2 \mathrm{~h}$ with agitation. The streptavidin agarose beads specifically bind to the DNA probe. The mixture was pelleted by centrifugation at $700 \mathrm{x} \mathrm{g}$ at $4^{\circ} \mathrm{C}$ for $10 \mathrm{~min}$ and washed with PBS three times. The bound proteins were analyzed by subsequent western blotting.

Western blotting. Cells were lysed with radioimmunoprecipitation assay buffer which containing a mixture of protease inhibitors (Santa Cruz Biotechnology, Inc.). Bicinchoninic acid protein assay kit (Invitrogen; Thermo Fisher Scientific, Inc.) was used to determine the protein concentration of the cells. A total of $40 \mu \mathrm{g}$ of protein was electrophoresed and separated by $10 \%$ SDS-PAGE. The protein was transferred to a polyvinylidene fluoride (PVDF) membrane (Invitrogen; Thermo Fisher Scientific, Inc.). Subsequent to blocking the PVDF membrane using blocking buffer for $1 \mathrm{~h}$ at $37^{\circ} \mathrm{C}$, the blots were incubated with the anti-c-Myc (dilution, 1:100), anti-Sirt1 (dilution, 1:200), anti-p53 (dilution, 1:500) and anti-GAPDH (dilution, 1:500) antibodies as previously described, at $4^{\circ} \mathrm{C}$ for $12 \mathrm{~h}$. The PVDF membrane was then washed with TBS-Tween-20 buffer and incubated with horseradish peroxidase-conjugated anti-mouse IgG (dilution, 1:100; cat. no. sc-358914; Santa Cruz Biotechnology, Inc.) for $1 \mathrm{~h}$ at $37^{\circ} \mathrm{C}$. An enhanced chemiluminescence kit (Beyotime Institute of Biotechnology, Haimen, China) and gel imaging system (Shanghai Furi Science and Technology Co., Ltd., Shanghai, China) were used for immune complex detection, according to the manufacturer's protocol. GAPDH was used as the internal control protein. The bands were analyzed by densitometry using Image $\mathbf{J}$ software version 1.47 (National Institutes of Health, Bethesda, MD, USA).

Statistical analysis. All the statistical analyses were performed using SPSS version 17.0 (SPSS, Inc., Chicago, IL, USA). All analyses were repeated three times and the data are expressed as the mean \pm standard deviation. The comparison between the two groups was performed using Student's t-test. The comparison between the three groups was based on one-way analysis of variance. When the difference was significant, the SNK-q test method was used to compare between groups. $\mathrm{P}<0.05$ was considered to indicate a statistically significant difference.

\section{Results}

c-Myc upregulates the protein expression level of Sirt1 in 553 deficient cells. In order to determine the effect of c-Myc on Sirt1 expression level, c-Myc was overexpressed in various cells lines, including K562, H1299, U2OS and 293A. Overexpression of c-Myc significantly increased the protein expression level of Sirt1 in K562 and H1299 cells compared with the control (Fig. 1A; P<0.05); however, an increase of Sirt1 protein expression level was not observed accompanying c-Myc overexpression in U2OS and 293A cells. K562 and H1299 cells are p53 deficient, whereas in U2OS and 293A cells, p53 is expressed (14-16). This implied the involvement of p53 in the c-Myc regulation of Sirtl expression level.
To further confirm the roles of p53 and c-Myc on Sirt1 expression regulation, c-Myc was overexpressed in p53 wild type and knockout MEF/3T3 cells. In agreement with a previous study (17), the protein expression level of Sirt1 was markedly increased in p53 knockout MEF/3T3s compared with wild-type MEF/3T3s (Fig. 1B). As expected, enforced expression of c-Myc significantly upregulated the protein expression level of Sirt1 in $\mathrm{p} 53^{-/} \mathrm{MEF} / 3 \mathrm{~T} 3$ cells $(\mathrm{P}<0.05)$, but had no effects on Sirt1 expression level in wild type MEF/3T3 cells (Fig. 1B). In addition, c-Myc inhibitor 10058-F4 treatment largely reduced Sirt1 protein expression level in p53 ${ }^{-/} \mathrm{MEF} / 3 \mathrm{~T} 3$ and $\mathrm{p} 53^{--}$HCT116 cells $(\mathrm{P}<0.05$ and $\mathrm{P}<0.01$ with 25 and $50 \mu \mathrm{M} 10058-\mathrm{F} 4$, respectively, for the two cell lines) but not in $\mathrm{p} 53^{+/+} \mathrm{MEF} / 3 \mathrm{~T} 3$ and $\mathrm{p} 53^{+/+}$HCT116 cells (Fig. 1C). The aforementioned results suggest that c-Myc may function as a positive regulator of Sirt1 expression level, whereas p53 inhibited regulation.

c-Myc upregulates the mRNA expression level of Sirt1 in p53 deficient cells. To further explore the regulatory effect of c-Myc on Sirt1 transcription and the underlying mechanism of $\mathrm{p} 53$ involvement in c-Myc upregulation to Sirt1, the change of Sirt1 mRNA expression level was examined upon experimental modulation of c-Myc activity in $\mathrm{p} 53$ wild type or deficient cells by qPCR. Overexpression of c-Myc resulted in an increased mRNA expression level of Sirt1 in p53 deficient K562 cells (Fig. 2A, left panel). Consistently, 10058-F4 treatment induced downregulation of Sirt1 mRNA expression level in K562 cells (right panel). Conversely, neither overexpression of c-Myc or 10058-F4 treatment altered the mRNA expression level of Sirt1 in 293A cells, which express p53 (Fig. 2B). Subsequently, the present study detected the mRNA expression level of Sirt1 in primary $\mathrm{MEF} / 3 \mathrm{~T} 3$ cells from $\mathrm{p} 53$ wild type or knockout mice. The mRNA expression level of Sirt1 was significantly elevated in $\mathrm{p} 53^{-/-} \mathrm{MEF} / 3 \mathrm{~T} 3 \mathrm{~s}$ and overexpression of c-Myc induced a further increase of Sirt1 mRNA expression level in the absence of p53 (Fig. 2C, left panel). Furthermore, 10058-F4 impaired the mRNA expression level of Sirt1 in p53 knockout, but not in p53 wild type MEF/3T3 (Fig. 2C, right panel). The results indicated that p53 may affect the transactivation ability of c-Myc on the Sirt1 promoter.

p53 inhibits the binding of c-Myc on the Sirt1 promoter. To further elucidate the mechanism underlying p53-mediated inhibition of the transactivation ability of c-Myc on Sirt1, the present study first predicted putative c-Myc binding elements in the human Sirtl promoter using an online program (rVISTA2.0). A total of three potential c-Myc binding elements, consensus sequence CACGTG, were identified in the human Sirt1 promoter 5'-flanking region, and were named E1, E2 and E3, respectively (Fig. 3A). However, E1 was not conserved across species when comparing the human with mouse and cow counterparts. In contrast, E2 and E3 are highly conserved among various types of species. The present study hypothesized that E2 and E3, but not E1, were the response elements for c-Myc.

To verify this possibility, the dual luciferase assay was performed to evaluate the activity of truncated deletion constructs of the Sirt1 promoter, $-2852,-200$ and -102 . Truncation of the promoter to -102 significantly decreased the baseline activity of 
A

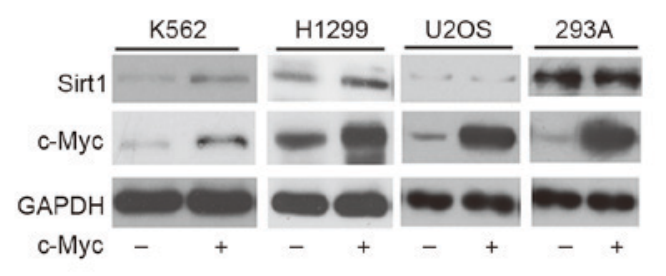

B

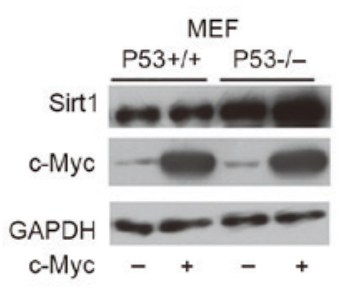

C

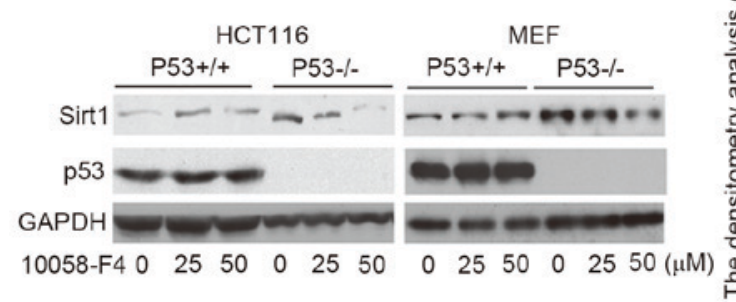

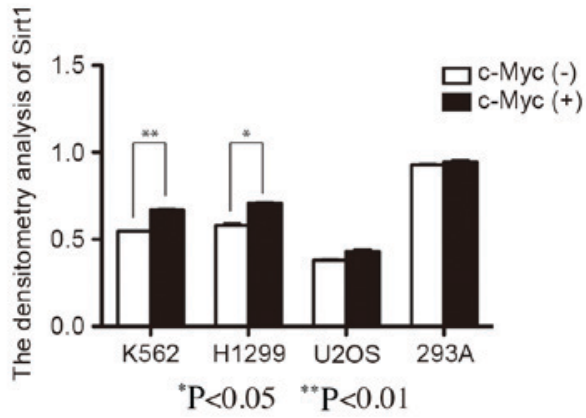

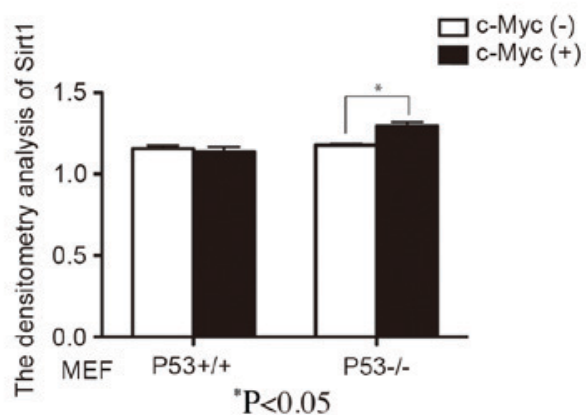

" $\mathrm{P}<0.05$

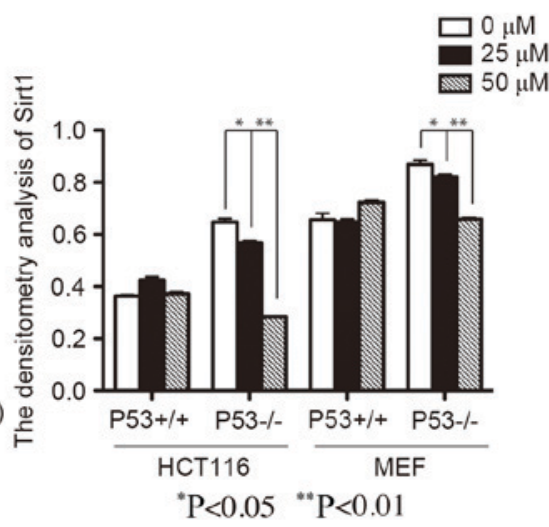

Figure 1. p53 inhibits the upregulation of Sirt1 protein expression level mediated by c-Myc. (A) Left panel presents K562, H1299, U2OS and 293A cells transfected with c-Myc expression vectors. Right panel presents the quantitation of Sirt1 protein expression levels from K562, H1299, U2OS and 293A cells using GAPDH expression as the internal control (Student's t-test; $n=3 ; \mathrm{P}<0.05$ ). Error bars represent the mean \pm standard error. (B) Left panel presents p53 wild type or knockout MEF/3T3 transfected with c-Myc expression vectors. Right panel presents the quantitation of Sirt1 protein expression levels from p53 wild type or knockout MEF/3T3 using GAPDH expression level as the internal control (Student's t-test; $\mathrm{n}=3$; $\mathrm{P}<0.05$ ). (C) Left panel presents $\mathrm{p}^{+/+}$or $\mathrm{p} 53^{-/-} \mathrm{MEF} / 3 \mathrm{~T} 3$ and HCT116 cells treated with 10058-F4 with indicated concentrations for $24 \mathrm{~h}$. Right panel presents the quantitation of Sirt1 protein expression levels from p53 ${ }^{+/+}$ or $\mathrm{p}^{-/-} \mathrm{MEF} / 3 \mathrm{~T} 3$ and HCT116 cells using GAPDH expression level as the internal control (SNK-q test; $\mathrm{P}<0.05$ ). Cell lysates of A, B and C were analyzed by western blotting. Sirt1, sirtuin 1 ; p53, tumor protein $\mathrm{p} 53$. ${ }^{*} \mathrm{P}<0.05 ;{ }^{* *} \mathrm{P}<0.01$.

the promoter in the control group compared with the full length Sirt1 promoter (-2852; Fig. 3B) whereas truncation to -200 did not, indicating that the proximal region of the promoter $(-200$ to -102) is important for promoter activity. Overexpression of c-Myc significantly upregulated full length and -200 truncated Sirtl promoter activity up to 2-fold compared with the control (Fig. 3B; $\mathrm{P}<0.05)$; however, deletion of the proximal region $(-200$ to -102) abolished c-Myc dependent transactivation (Fig. 3B). These results suggested that c-Myc transactivates the Sirt1 promoter via E2 or E3 but not E1.

To further define the exact response element of c-Myc on human Sirt1 promoter, E2 or E3 point mutants of the Sirt1 promoter were generated, where the individual c-Myc binding sites were mutated. As presented in Fig. $3 \mathrm{C}(\mathrm{P}<0.05)$, the point mutation of E2 markedly reduced the induction of Sirt1 promoter activity by c-Myc compared with the truncated wild-type Sirt1 promoter -200, whereas E3/mut was activated by c-Myc to a similar level compared with the truncated wild-type Sirt1 promoter -200. The results suggested that E2 may be the response element for c-Myc.

Subsequently, the results were confirmed by DNA mediated precipitation assay. Biotinylated double strand oligonucleotide 222 (Sirt1 promoter sequence containing E2) or 276 (50 bp upstream of 222, used as the negative control) were incubated with HeLa nuclear extracts and streptavidin agarose beads slurry and the precipitates were analyzed by western blotting. As presented in Fig. 3D, c-Myc bound to the probe 222 but not 276, whereas the negative control GAPDH did not 

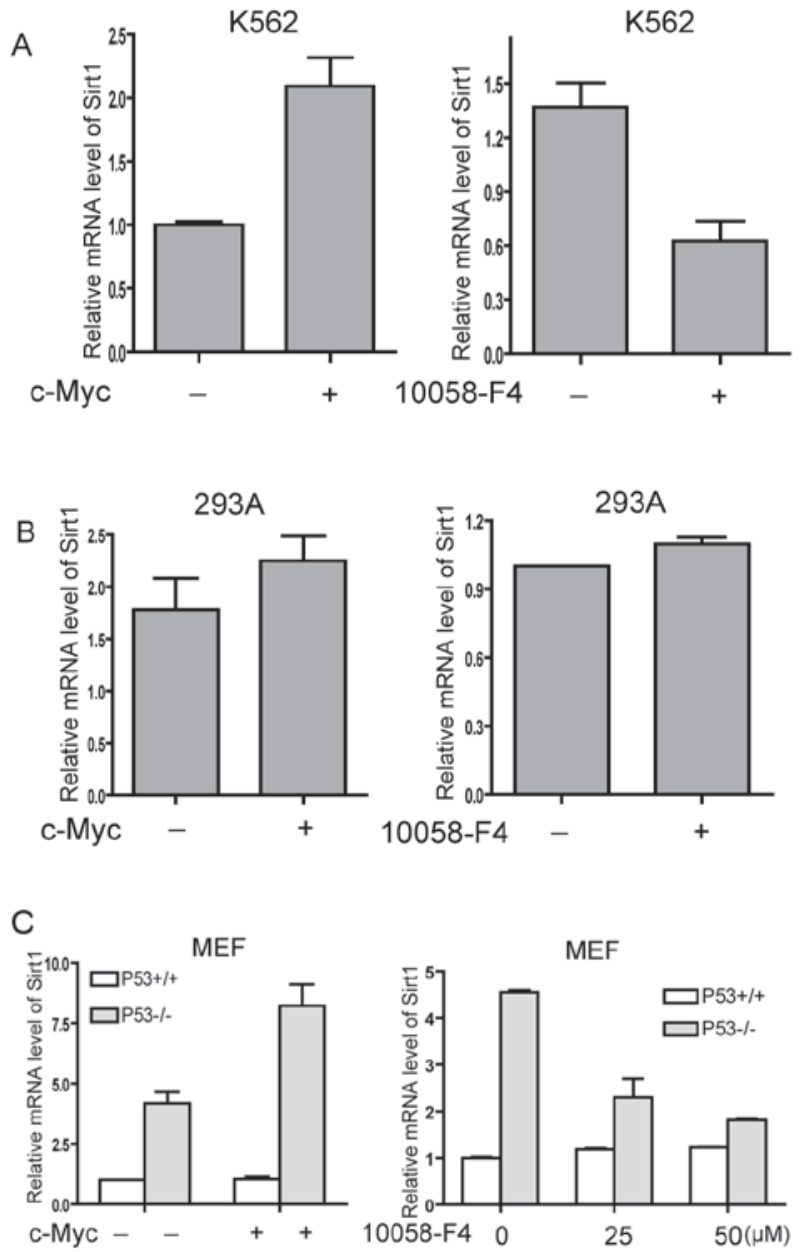

Figure 2. p53 inhibits the upregulation of Sirt1 mRNA level mediated by c-Myc. (A) K562 cells were transfected with c-Myc expression vectors or treated with 10058-F4. (B) 293A cells were transfected with c-Myc expression vectors or treated with 10058-F4. (C) p53 wild type or knockout MEF/3T3 were transfected with c-Myc expression vectors or treated with 10058-F4 RNA was extracted from cells in A, B and C, and analyzed by quantitative polymerase chain reaction. Sirt1, sirtuin 1; 553 , tumor protein $\mathrm{p} 53$.

bind to the two probes. This further demonstrated that E2 was the binding site of c-Myc.

Of note, the E2 site forms part of the element previously identified to respond to p53 (10). The overlapping two elements may serve a role in the differential regulatory effects of c-Myc on Sirt1 in the absence or presence of p53. In the present study, c-Myc and p53 expressing plasmids (-200) were co-transfected together with Sirt1 promoter reporter plasmids into p53 deficient H1299 cells. As presented in Fig. 3E, overexpression of p53 decreased the Sirt1 promoter activity and abolished the c-Myc-dependent activation of the promoter, which suggested that c-Myc-dependent upregulation of Sirt1 was inhibited by p53 at the transcriptional level.

Based on the aforementioned results, it was hypothesized that p53 and c-Myc share $\geq 1$ response element on the Sirt1 promoter. The binding of p53 may block the recruitment of c-Myc onto its response element. Therefore, a ChIP assay was performed with K562 and 293A cells. The results revealed that the Sirt1 promoter fragment containing E2 co-precipitated with endogenous c-Myc in p53 deficient K562 cells but not in 293A cells expressing p53 (Fig. 3F). In agreement with the luciferase assay results, E1 was not present in the DNA precipitate of c-Myc in K562 or 293A cells. The hTERT promoter was amplified as a positive control. Taken together, these results suggested that p53 inhibited the binding of c-Myc on the Sirt1 promoter, thus blocking the upregulation of Sirt1 expression level mediated by c-Myc.

\section{Discussion}

Previous studies have revealed that Sirtl expression is tightly regulated by certain transcriptional factors, including p53, HIC1 and E2F1 $(2,10,11)$. The present study demonstrated that Sirt1 transcription was induced by c-Myc when p53 was deficient. The response element for c-Myc induction was co-located with the p53 binding site of the Sirt1 promoter. Therefore, p53 inhibited the upregulation of Sirt1 expression mediated by c-Myc by blocking the binding of c-Myc on the Sirt1 promoter.

Tumor suppressor p53 serves a critical role in suppression of cell growth and proliferation, whereas the oncoprotein c-Myc conversely regulates p53-associated physiological and pathological processes. It is known that there is a negative association between p53 and c-Myc (18). However, the precise mechanism underlying the repression of p53 and c-Myc has not been fully understood. Early reports suggested that p53 directly suppressed the transcription of c-Myc (19), whereas c-Myc inhibited p53 expression level via p19ARF, which forms a feedback loop (20). Another post-transcriptional mechanism underlying p53 repression of c-Myc involves microRNA (miR) -145 (21). p53 induced miR-145 expression and subsequently repressed c-Myc at the protein level (21). The results of the present study suggest a new mechanism underlying p53 repression of c-Myc function; p53 and c-Myc compete for binding to the same response element, and inversely regulate the expression level of Sirt1. p53 appears to have a stronger affinity to the binding site since the present study didn't detect the binding of c-Myc on the Sirt1 promoter in p53 expressing cells. It's noteworthy that Sirt1 is not the first target identified to be regulated by p53 and c-Myc in a similar manner. It is reported that optimal induction of cyclinB1 promoter by c-Myc only occurs when p53 is concurrently inactivated (22).

Deregulation of c-Myc oncogene or loss of p53 activity occurs frequently in certain types of human cancer (23). The results of the present study suggested that c-Myc overexpression affects the transactivation ability of c-Myc on the Sirt1 promoter in p53 deficient cells, elevating the expression level of Sirt1. The combination of c-Myc overexpression and p53 loss of function may contribute to the elevated expression level of Sirt1 in tumor cells, as cells with high expression levels of Sirt1, including AML, squamous cell carcinoma and diffuse large B-cell lymphoma, demonstrated c-Myc overexpression (24-26) and p53 loss of function (27). The regulation of Sirt1 expression level by c-Myc and p53 prompted the present study to consider Sirt1 as a target to mediate the oncogenic function of c-Myc.

Taken together, the results of the present study demonstrated that Sirt1 expression level was directly but conversely regulated by c-Myc and p53. p53 inhibited the activation of the Sirt1 promoter by blocking c-Myc recruitment on the Sirt1 
A

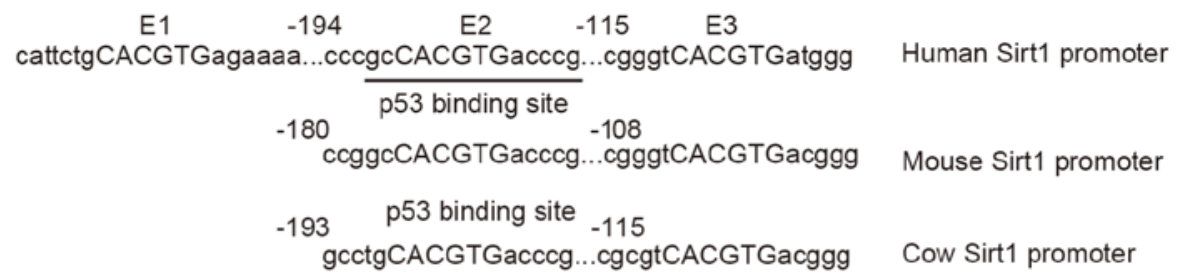
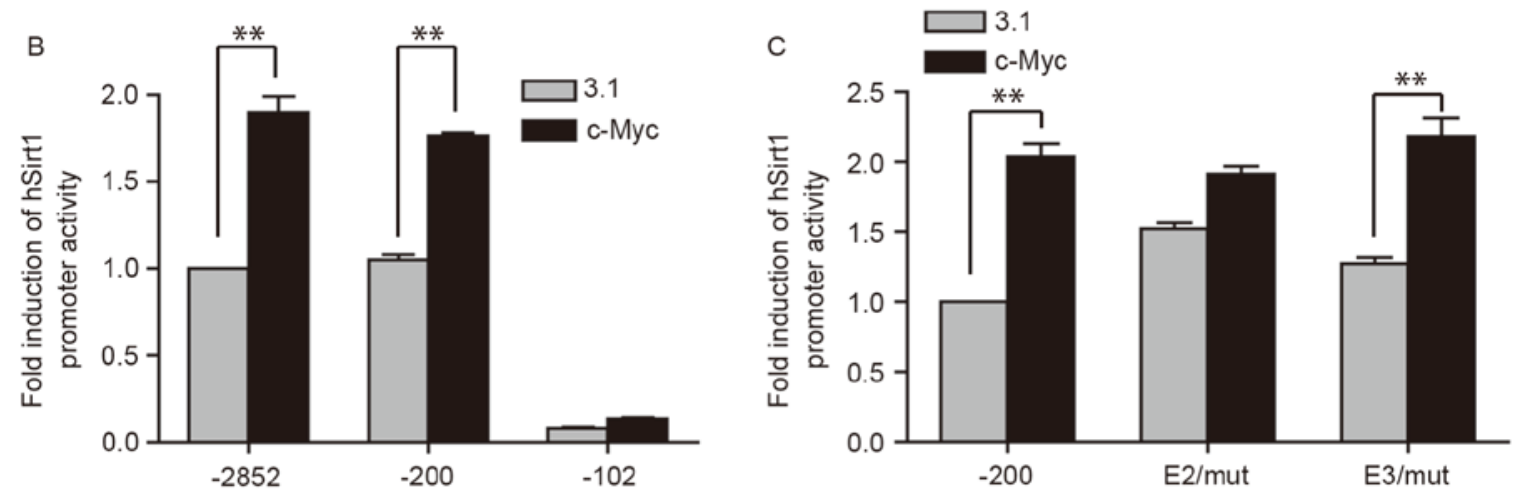

D
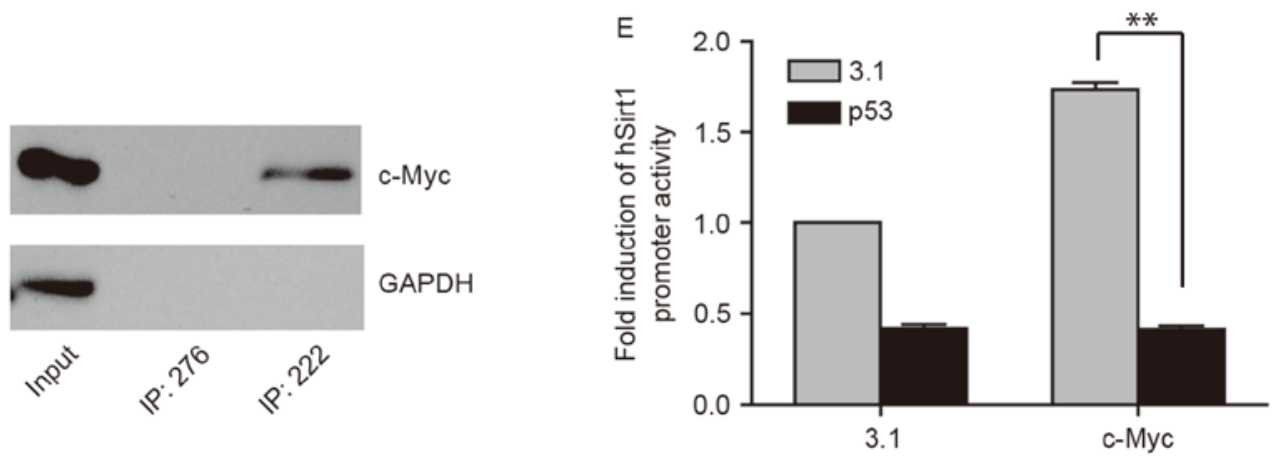

$\mathrm{F}$

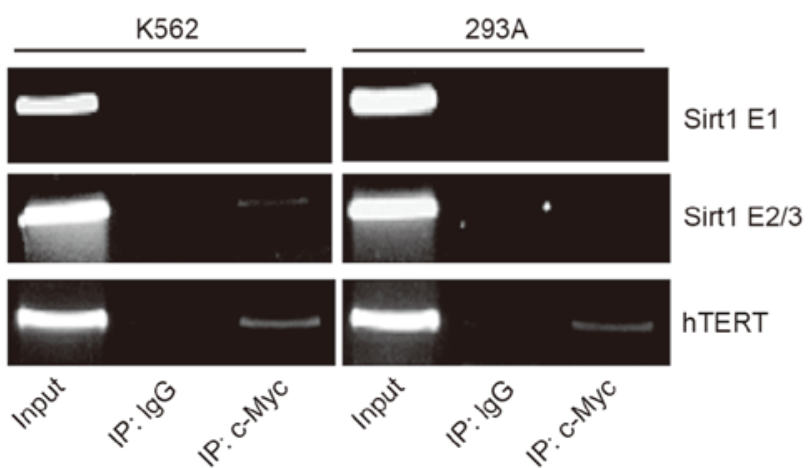

Figure 3. p53 blocks the binding of c-Myc to the Sirt1 promoter. (A) Alignment of the proximal core region of human, mouse and cow Sirt1 promoter sequences. The underlined bases indicate the p53 binding site and capital bases indicate the putative c-Myc binding sites. (B) Human Sirt1 promoter reporter plasmids containing 2852,200 or 102 bp upstream of the transcription start site were separately co-transfected with pcDNA 3.1 or c-Myc expression vectors into H1299 cells for 36 h. (C) H1299 cells were co-transfected with wild type (-200) or mutated Sirt1 promoter reporter plasmids (E2/mut or E3/mut) with c-Myc expression vector. (D) HeLa nuclear extracts were incubated with biotinylated oligonucleotides 222 containing E2 box of human Sirt1 promoter or 276 (50 bp upstream of 222) and streptavidin-agarose beads. The precipitated complexes were analyzed by western blotting. (E) H1299 cells were co-transfected with Sirt1 promoter reporter plasmids with c-Myc and p53 expression vectors as indicated. The relative luciferase activities of $\mathrm{B}, \mathrm{C}$ and $\mathrm{E}$ are presented as the mean \pm standard deviation of triplicate samples and were representative of three independent experiments. (F) Chromatin immunoprecipitation assay was performed in K562 and 293A cells to detect the recruitment of c-Myc on the Sirt1 promoter E2 and E1 sites. The hTERT promoter was used as positive control. Sirt1, sirtuin 1; bp, base pair; mut, mutant; hTERT, human telomerase reverse transcriptase; p53, tumor protein p53. ${ }^{*} \mathrm{P}<0.05 ;{ }^{* *} \mathrm{P}<0.01$.

promoter. The present study revealed a novel network that regulates the expression of Sirtl and further elucidated the counterbalance of a tumor suppressor and promoter.

\section{Acknowledgements}

The present study was supported by the National Natural 
Science Foundation of China (grant no. 81402319) and the Beijing Nova Program (grant no. Z161100004916133).

\section{References}

1. Liu TF and McCall CE: Deacetylation by SIRT1 reprograms inflammation and cancer. Genes Cancer 4: 135-147, 2013.

2. Wang C, Chen L, Hou X, Li Z, Kabra N, Ma Y, Nemoto S, Finkel T, Gu W, Cress WD and Chen J: Interactions between E2F1 and SirT1 regulate apoptotic response to DNA damage. Nat Cell Biol 8: 1025-1031, 2006.

3. McCall CE, El Gazzar M, Liu T, Vachharajani V and Yoza B: Epigenetics, bioenergetics, andmicroRNA coordinate gene-specific reprogramming during acute systemic inflammation. J Leukoc Biol 90: 439-446, 2011.

4. Hida Y, Kubo Y, Murao K and Arase S: Strong expression of a longevity-related protein, SIRT1, in Bowen's disease. Arch Dermatol Res 299: 103-106, 2007.

5. Huffman DM, Grizzle WE, Bamman MM, Kim JS, Eltoum IA, Elgavish A and Nagy TR: SIRT1 is significantly elevated in mouse and human prostate cancer. Cancer Res 67: 6612-6618, 2007.

6. Hennig D, Müller S, Wichmann C, Drube S, Pietschmann K, Pelzl L, Grez M, Bug G, Heinzel T and Krämer $\mathrm{OH}$ Antagonism between granulocytic maturation and deacetylase inhibitor-induced apoptosis in acute promyelocytic leukaemia cells. Br J Cancer 112: 329-337, 2015.

7. Jang KY, Hwang SH, Kwon KS, Kim KR, Choi HN, Lee NR, Kwak JY, Park BH, Park HS, Chung MJ, et al: SIRT1 expression is associated with poor prognosis of diffuse large B-cell lymphoma. Am J Surg Pathol 32: 1523-1531, 2008.

8. Shah ZH, Ahmed SU, Ford JR, Allison SJ, Knight JR and Milner J: A deacetylase-deficient SIRT1 variant opposes full-length SIRT1 in regulating tumor suppressor p53 and governs expression of cancer-related genes. Mol Cell Biol 32: 704-716, 2012.

9. Vahtola E, Louhelainen M, Forstén H, Merasto S, Raivio J, Kaheinen P, Kytö V, Tikkanen I, Levijoki J and Mervaala E: Sirtuin1-p53, forkhead box O3a, p38 and post-infarct cardiac remodeling in the spontaneously diabetic Goto-Kakizaki rat. Cardiovasc Diabetol 9: 5, 2010.

10. Nemoto S, Fergusson MM and Finkel T: Nutrient availability regulates SIRT1 through a forkhead-dependent pathway. Science 306: 2105-2108, 2004.

11. Chen WY, Wang DH, Yen RC, Luo J, Gu W and Baylin SB Tumor suppressor HIC1 directly regulates SIRT1 to modulate p53-dependent DNA-damage responses. Cell 123: 437-448, 2005 .

12. Mao B, Zhao G, Lv X, Chen HZ, Xue Z, Yang B, Liu DP and Liang CC: Sirt1 deacetylates c-Myc and promotes c-Myc/Max association. Int J Biochem Cell Biol 43: 1573-1581, 2011.

13. Livak KJ and Schmittgen TD: Analysis of relative gene expression data using real-time quantitative PCR and the 2(-Delta Delta C(T)) method. Methods 25: 402-408, 2001.

14. Yamaguchi H, Woods NT, Piluso LG, Lee HH, Chen J, Bhalla KN, Monteiro A, Liu X, Hung MC and Wang HG: P53 acetylation is crucial for its transcription-independent proapoptotic functions. J Biol Chem 284: 11171-11183, 2009.

15. Rothmann T, Hengstermann A, Whitaker NJ, Scheffner M and zur Hausen H: Replication of ONYX-015, a potential anticancer adenovirus, is independent of p53 status in tumor cells. J Virol 72: 9470-9478, 1998.
16. D'Orazi G, Cecchinelli B, Bruno T, Manni I, Higashimoto Y, Saito S, Gostissa M, Coen S, Marchetti A, Del Sal G, et al: Homeodomain-interacting protein kinase-2 phosphorylates p53 at Ser 46 and mediates apoptosis. Nat Cell Biol 4: 11-19, 2002.

17. Wang S, Song P and Zou MH: Inhibition of AMP-activated protein kinase $\alpha(\mathrm{AMPK} \alpha)$ by doxorubicin accentuates genotoxic stress and cell death in mouse embryonic fibroblasts and cardiomyocytes: Role of p53 and SIRT1. J Biol Chem 287: 8001-8012, 2012.

18. Xie Y, Bulbul MA, Ji L, Inouye CM, Groshen SG, Tulpule A O'Malley DP, Wang E and Siddiqi IN: P53 expression is a strong marker of inferior survival in de novo diffuse large B-cell lymphoma and may have enhanced negative effect with MYC coexpression: A single institutional clinicopathologic study. Am J Clin Pathol 141: 593-604, 2014.

19. Ho JS, Ma W, Mao DY and Benchimol S: P53-dependent transcriptional repression of c-myc is required for G1 cell cycle arrest. Mol Cell Biol 25: 7423-7431, 2005

20. Ta VB, de Bruijn MJ, ter Brugge PJ, van Hamburg JP, Diepstraten HJ, van Loo PF, Kersseboom R and Hendriks RW: Malignant transformation of Slp65-deficient pre-B cells involves disruption of the Arf-Mdm2-p53 tumor suppressor pathway. Blood 115: 1385-1393, 2010.

21. Sachdeva M, Zhu S, Wu F, Wu H, Walia V, Kumar S, Elble R, Watabe K and Mo YY: P53 represses c-Myc through induction of the tumor suppressor miR-145. Proc Natl Acad Sci USA 106: 3207-3212, 2009

22. Molinuevo R, Freije A, de Pedro I, Stoll SW, Elder JT and Gandarillas A: FOXM1 allows human keratinocytes to bypass the oncogene-induced differentiation checkpoint in response to gain of MYC or loss of p53. Oncogene 36: 956-965, 2017.

23. Tanaka H, Tamura A, Sekai M, Hamazaki Y and Minato N: Increased c-Myc activity and DNA damage in hematopoietic progenitors precede myeloproliferative disease in Spa-1-deficiency. Cancer Sci 102: 784-791, 2011.

24. Li L, Osdal T, Ho Y, Chun S, McDonald T, Agarwal P, Lin A, Chu S, Qi J, Li L, et al: SIRT1 activation by a c-MYC oncogenic network promotes the maintenance and drug resistance of human FLT3-ITD acute myeloid leukemia stem cells. Cell Stem Cell 15: 431-446, 2014

25. Savage KJ, Johnson NA, Ben-Neriah S, Connors JM, Sehn LH, Farinha P, Horsman DE and Gascoyne RD: MYC gene rearrangements are associated with a poor prognosis in diffuse large B-cell lymphoma patients treated with R-CHOP chemotherapy. Blood 114: 3533-3537, 2009.

26. Liu Y, Gong LP, Dong XL and Liu HG: Detection of C-MYC oncogene translocation and copy number change in the normal-dysplasia-carcinoma sequence of the larynx by fluorescence in situ hybridization. Diagn Cytopathol 41: 515-519, 2013.

27. Balz V, Scheckenbach K, Götte K, Bockmühl U, Petersen I and Bier H: Is the p53 inactivation frequency in squamous cell carcinomas of the head and neck underestimated? Analysis of p53 exons 2-11 and human papillomavirus 16/18 E6 transcripts in 123 unselected tumor specimens. Cancer Res 63: 1188-1191, 2003. 